\title{
Über die Schwankungen der abiotischen Faktoren in der Elbmündung bei Cuxhaven
}

\author{
HEINRICH KÜHL \\ Institut für Küsten- und Binnenfischerei \\ der Bundesforschungsanstalt für Fischerei, Hamburg
}

\begin{abstract}
On the fluctuations of abiotic factors in the Elbe estuary near Cuxhaven. The changes of abiotic factors, especially salinity, water temperature, $\mathrm{O}_{2}$-content, $\mathrm{pH}$ and alcalinity in the Elbe river near Cuxhaven are documented. Long-term salinity fluctuations depend on the water outflow of the Elbe. Short-term salinity changes are caused by the tides. During the period from 1949 to 1953 and again in 1959, salinity was high (above 20\% in summer). From 1954 to 1958 , as well as in 1961, salinity was low, at times considerably below $15 \%$. At high tide with its increasing salinity, plankton samples contain more copepods and larvae of balanids and spionids since the maximum population density of these forms occurs in the polyhalinicum at the light-ship "Elbe 3". If the conditions are favorable, the larvae can settle and start a new population. In a small locality near the "Alte Liebe" pier, marked off by two jetties, the salinity is always somewhat higher than it is in the river Elbe. In this higher salinity, populations of Coryne sarsi as well as young Asterias rubens were found in all years characterized by increased salinities (monthly average about 15 to $20 \%$ ). In years with higher salinities Teredo navalis caused increased deterioration of timber work. Small water pools in the mud flat area showed profound changes in water temperature, salinity and $\mathrm{pH}$. Their $\mathrm{O}_{2}$ content is very high due to the photosynthesis of diatoms, reaching saturation values of $320 \%$. These extremes disappear quickly as the water returns at high tide.
\end{abstract}

\section{EINLEITUNG}

Als Kirchenpauer in den Jahren 1859-62 den Bewuchs der Seetonnen in der Elbmïndung von See bis St. Margarethen, zwischen Brunsbüttel und Glückstadt, untersuchte, fand er eine ganz charakteristische, also nicht zufällige Verteilung der Hydroiden-Besiedlung vor. Von See her folgten einander Sertularia argentea $(=\mathrm{cu}$ pressina), Tubularia larynx, Laomedea gelatinosa und Cordylopbora albicola ( $=C$. caspia $f$. albicola). Auf Grund dieser Befunde, denen sich auch andere Organismen zuordnen ließen, teilte KIRCHENPAUER die Elbmündung in die Regionen I-IV auf. Er fragte sich dann, welche Faktoren diese bemerkenswerte Verbreitung verursachten und diskutierte die Bedeutung des Substrates bzw. des Flußbodens, der Wassertiefe, der Wassertemperatur, der Reinheit (Trübung) des Wassers, des Wellenschlages und der Strömung. Alle diese Faktoren waren zwar nicht bedeutungslos, sie reichten aber für die Erklärung nicht aus. „Nach all diesem wird es wahrscheinlich, daß nur allein der größere oder geringere Salzgehalt es ist, welcher entscheidend auf den spezifischen 
Charakter der Vegetation an den Seetonnen wirkt ..." „Die Abnahme des Salzgehaltes von der Mündung stromaufwärts oder das Mischungsverhältnis zwischen See- und Flußwasser ist an demselben Ort der Unterelbe fortwährend veränderlich. Zunächst kommt die Menge des oberen Zuflusses dabei wesentlich in Betracht. Dieser Zufluß pflegt im Frühjahr größer zu sein als im Herbst und daher erstreckt sich der Salzgehalt des Elbwassers auch im Herbst weiter stromaufwärts als im Frühjahr. Einen wesentlichen Einfluß auf den Salzgehalt des Wassers in den unteren Flußregionen übt auch die tägliche Ebbe und Fluth; kräftige Fluthen, welche durch westliche Winde über die gewöhnliche Höhe getrieben werden, erhöhen den Salzgehalt des Elbwassers, während derselbe bei schwachen Fluthen abnimmt."

Anhand von biologischen Beobachtungen weist KIRCHENPAUER auch darauf hin, daß der Salzgehalt in der Tiefe größer ist als an der Oberfläche als "natürliche Folge der größeren Schwere...". Bei den mehrjährigen Untersuchungen bemerkte KrRCHENPAUER auch die Unterschiede in der Besiedlung in den einzelnen Jahren: „wie auffallend der Charakter der Vegetation nicht bloß nach den Lokalitäten, sondern an derselben Lokalität nach den Jahren verschieden ist..., denn die Bewohnerschaft ist einem Wechsel unterworfen, dessen Gesetze noch nicht ermittelt sind ..., sondern auch in dem Sinne, daß dieselben Arten in einem Jahre da sind, in einem anderen fehlen und in einem folgenden wiederkommen. DAHL (1892) konnte bei seinen Untersuchungen iber die Tierwelt der Unterelbe die KirchenPauERschen Ergebnisse im wesentlichen bestätigen. Bei einem Vergleich der II. Region KirChEnPauERs mit der Neustädter Bucht in der Ostsee fand DAHL hier die doppelte Anzahl von Arten wie im gleichen Salzgehaltsbereich der Unterelbe. Die Ursache hierfür sieht DAHL in der starken Strömung des Flusses. ThIEManN (1934) hingegen konnte zeigen, daß in bestimmten Brackwasserbereichen ein Minimum an Arten vorhanden ist, die sog. Verarmungszone. LÜNEBURG (1939) übertrug bei seinen hydrochemischen Untersuchungen der Elbmündung die Einteilung in haline Zonen von ReDEKE (1922) auf die Elbe, ohne Kenntnis der Kirchenpauerschen Arbeit. Beim Vergleich dieser beiden Arbeiten zeigt sich eine ausgezeichnete Ubereinstimmung zwischen den Regionen I-IV von KIRCHENPAUER und den halinen Zonen nach REDEKE, wobei noch einmal darauf hingewiesen sei, daß KrRCHENPAUER seine Einteilung anhand biologischer Befunde schuf. Auf weitere Arbeiten wird weiter unten im Zusammenhang noch eingegangen werden.

\section{UNTERSUCHUNGSERGEBNISSE}

Im folgenden sollen nun die Schwankungen einiger abiotischer Faktoren, wie Salzgehalt, Wassertemperatur, Sauerstoffgehalt, pH-Wert und Säurebindungsvermögen bei Cuxhaven, im Grenzgebiet zwischen Poly- und Mesohalinikum behandelt und kurz auf einige biologische Verhältnisse eingegangen werden.

Als biologisch wichtigster Faktor im Brackwassergebiet soll zunächst der Salzgehalt besprochen werden. Seine Schwankungen werden bedingt durch 1 . die Wasserfïhrung der Elbe, 2. die Gezeiten, 3. die Wetterverhältnisse, wie Wind-Richtung und -Stärke, Luftemperatur, Regen usw.

Der Einfluß der Oberwassermenge auf die Anderungen des Salzgehaltes läßt sich 
sehr gut aus Beobachtungen ablesen, die seit vielen Jahren täglich an der "Alten Liebe“ bei Cuxhaven bestimmt werden. In Abbildung 1 sind die Meßpunkte 1 (Alter Hafen, Versuchsstand 1) und 2 („Alte Liebe“, Versuchsstand 2) angegeben. Um den Einfluß der Gezeiten auf die Salzgehaltsänderungen auszuschalten, wurden in Abbildung 2

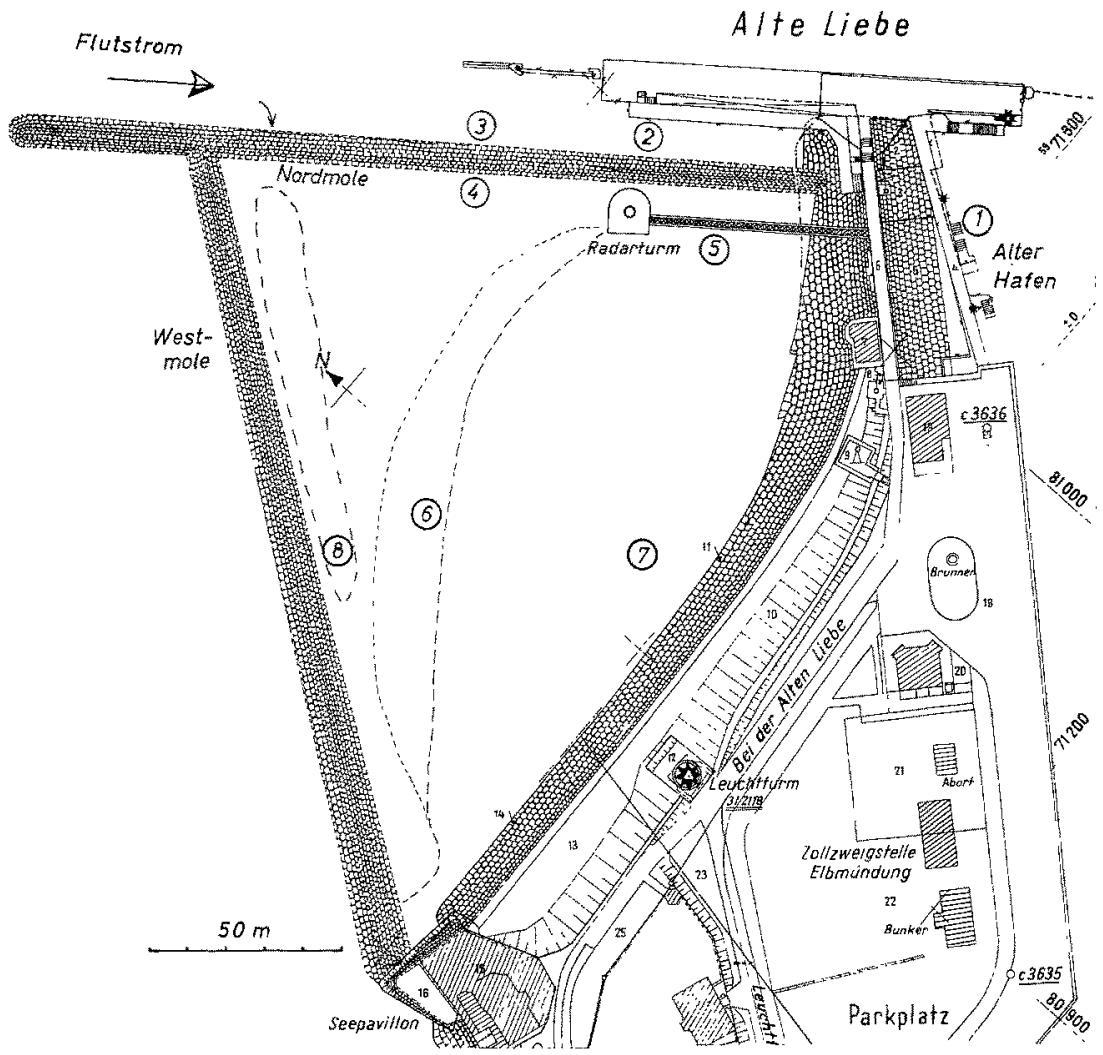

Abb. 1: Das Bollwerk "Alte Liebe", Alter Hafen und das "Molendreieck" in Cuxhaven mit Meßpunkten. Gestrichelte Linie: Wattgrenze im "Molendreieck"; feingestrichelte Linie bei sehr niedrigem Wasserstand. Strichpunktlinie: Rinne von 1-2 m Tiefe an der Westmole. Pfeile: Flutstrom

die Monatsmittel für Wassertemperatur und Salzgehalt an der Oberfläche für die Jahre von 1949-1962 eingetragen, außerdem noch die Abflußmenge der Elbe bei Artlenburg im Monatsmittel.

Deutlich zeigt sich nun, daß die Wassertemperatur im ganzen gesehen einen sehr regelmäßigen Verlauf hat, wenn sich auch im einzelnen in den verschiedenen Jahren deutliche Unterschiede bemerkbar machen, auf die an anderer Stelle näher eingegangen wurde (KüHL 1953-63). Anders verhält es sich mit den Salzgehalten, hier zeigen die Kurven einen sehr variablen Charakter. Sehr klar aber kommt die Abhängigkeit von der Oberwassermenge zum Ausdruck: ist diese gering, so nimmt der Salzgehalt zu und umgekehrt. 
In den Jahren 1949 bis 1953 und wieder 1959 ist der mittlere Salzgehalt recht hoch, er übersteigt im Sommer die 20\%-Linie; von 1954 bis 1958 sinken die Salzgehalte stark $\mathrm{ab}$, sie liegen zum 'Teil weit unter der $15 \%$-Linie. Wesentlich ist, daß diese Anderungen im allgemeinen langfristig sind, da sie von den Witterungsverhältnissen im großen Einzugsgebiet der Elbe abhängen. Wegen dieser längeren Einwirkungsdauer sind diese Salzgehaltsänderungen von besonderer Bedeutung für das biologische Geschehen.

Innerhalb dieser langfristigen Anderungen des Salzgehaltes bewirken die Gezeiten sehr schnelle, sich über Stunden erstreckende Veränderungen des Salzgehaltes, die aber

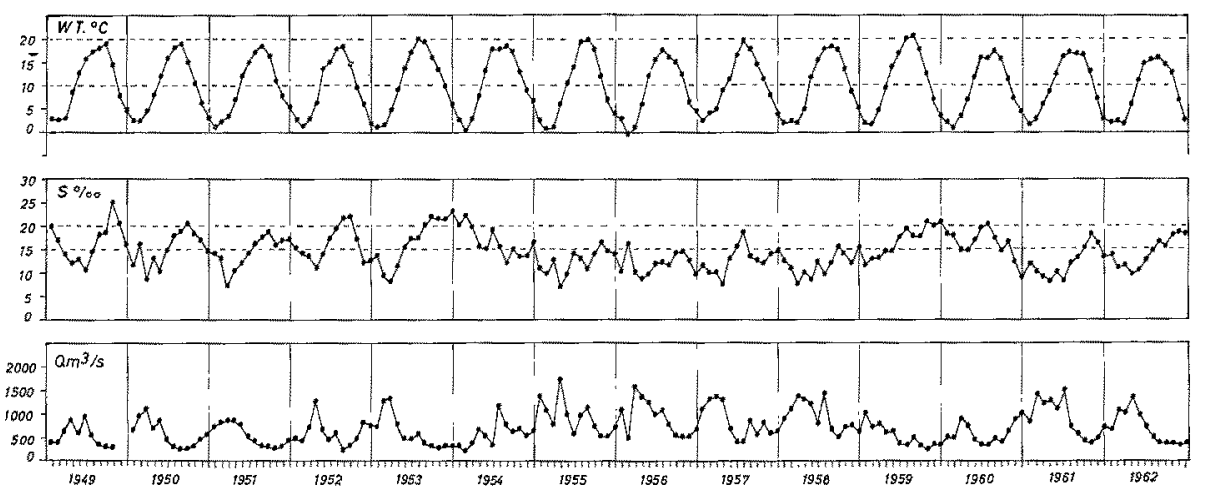

Ab́b. 2: Monatsmittel für Wassertemperatur (WT ${ }^{\circ} \mathrm{C}$ ), Salzgehalt (S\%) und Abflußmenge der Elbe bei Darchau $\left(\mathrm{Qm}^{3} / \mathrm{sec}\right)$ für 1949 bis 1962

oft ein beträchtliches Ausmaß erreichen können (LUckr 1953). Uber die Veränderungen der chemischen Faktoren und des Phytoplanktons während einer Tide wurde schon früher berichtet (BURSCHE, KüHL \& MANN 1958).

Am Bollwerk "Alte Liebe" befindet sich vor dem Deich ein durch Molen abgegrenztes Gebiet, das wir kurz „Molendreieck“ nennen wollen (Abb. 1), das über viele Jahre hin in verschiedener Weise untersucht wurde. Anhand einiger Beispiele soll gezeigt werden, in welchem Maße die abiotischen Faktoren schwanken, und wie schon durch geringfügige topographische Unterschiede bemerkenswerte Änderungen des Milieus zu bemerken sind. Das „Molendreieck" erhält seine Besonderheit dadurch, daß bei Ebbe das Wasser durch die Lücken der Nordmole abläut und das Becken ganz langsam trockenfällt, bis auf einen Rest an der Westmole. Die Menge des „Restwassers“ hängt von der Windlage ab. Wenn bei stärkeren östlichen Winden und Nipptiden der Wasserstand niedrig ist, ist auch die Menge des Restwassers gering (Abb. 1 gestrichelte Linien). Steigt bei Flut nun der Wasserspiegel, so läuft wiederum zunächst Seewasser durch die Lücken langsam in das Molendreieck, bei einem Pegelstand von $4,20 \mathrm{~m}$ (NN-5 m) wird die Mole überspiult, und in etwa 10 Minuten ist das ganze Molendreieck vollgelaufen. $\mathrm{Da}$ das Flutwasser die Nordmole zuerst am Westende wie in einem Wasserfall überspült, ist längs der Westmole ein 1-2 $\mathrm{m}$ tiefer Graben ausgespuilt worden. Das „Restwasser", das wie gesagt hier immer stehenbleibt, bewirkt, daß der Salzgehalt im Molendreieck immer um einige Promille höher liegt als im freien Strom. 
Von den zahlreichen Meßstellen, die wir benutzten, seien 7 charakteristische Meßpunkte ausgewählt (Abb. 1):

1 Alter Hafen, Versuchsstand 1

2 "Alte Liebe", Versuchsstand 2

3 Außerhalb der Nordmole im Strom

4 Unmittelbar innerhalb der Nordmole, in der Nähe einer kleinen Muschelbank

5 Eine größere Pfütze in der Nähe des Radarturmes

6 Am Wattrand des "Restwassers"

7 Eine größere Pfütze unterschiedlicher Ausdehnung mit Steinen, die von Fucus vesiculosus, Ulva lactuca, Enteromorpha spec., Porphyra umbilicalis u. a. bewadhsen sind.

Vor dem Uferwerk befinden sich zahlreiche Pfützen. Der Wattboden des Molendreieckes besteht aus Schlicksand, der zeitweilig stark mit Bodendiatomeen bedeckt ist. Um die Unterschiede im Salzgehalt selbst nahe beieinanderliegender Stellen zu zeigen, soll der Kurvenverlauf der Salzgehalte während einer Tide an den Punkten 1 (Alter Hafen), Punkt 2 ("Alte Liebe“) und Punkt 5 (Radarturm-Pfütze) nebeneinander dargestellt werden (Abb. 3). Die drei Meßpunkte liegen nur 30-90 m auseinander (Abb. 1). Zur Zeit um Tnw sind die Salzgehalte von Punkt 1 und 2 etwa gleich hoch; mit auflaufendem Wasser nimmt der Salzgehalt an der "Alten Liebe" (2) aber schneller zu, als im Alten Hafen (1), wobei beide Kurven aber der Pegelkurve nachlaufen. Die Kurve des Salzgehaltes für Punkt 5 - etwa $30 \mathrm{~m}$ von der "Alten Liebe entfernt - zeigt die Besonderheiten des Molendreiecks: bei abgelaufenem Wasser ist der Salzgehalt um etwa $6 \%$ höher. Wenn das auflaufende Wasser die Nordmole überspült (Abb. 3; 5.30 und 18.00 Uhr) erhalten die Salzgehalte sehr schnell gleiche Höhe, bei fallendem Wasser gehen sie wieder auseinander.

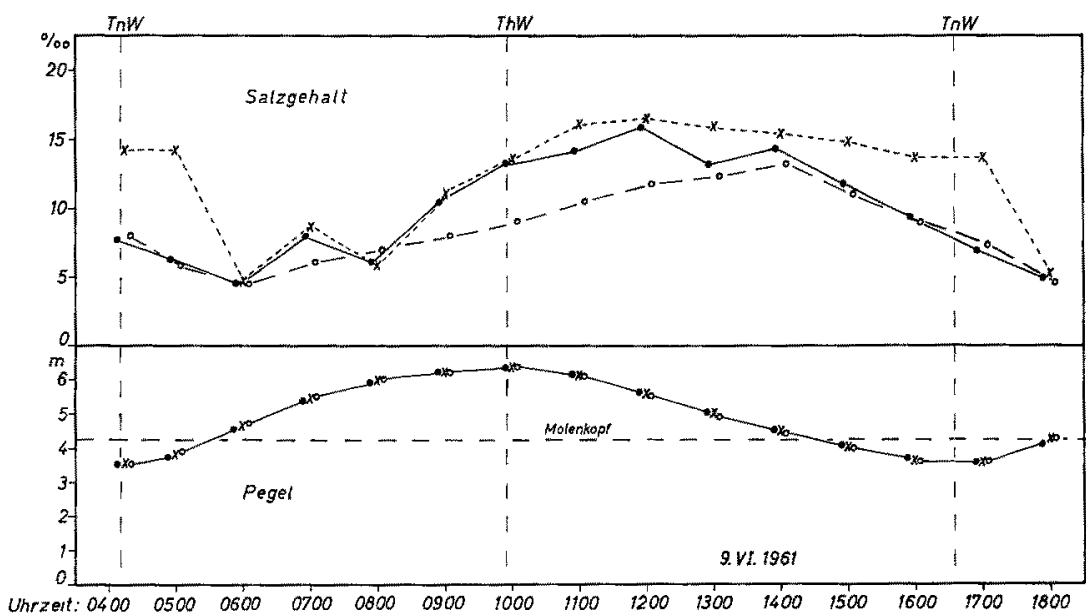

Abb. 3: Verlauf des Salzgehaltes über 14 Stunden (von Tnw-Tnw) an den Meßpunkten 1 (Alter Hafen) $0-0,2$ ("Alte Liebe") $\bullet-\bullet$ und 5 (Molendreieck" Radarturm) $\times$-..- $\times$ mit Wasserstandsangaben $(\mathrm{NN}-5 \mathrm{~m})$. Die gestrichelte Linie gibt an, wann die Nordmole vom Wasser überflutet wird 
Eine weitere Zusammenstellung gibt die Werte für Wassertemperatur, Salzgehalt, Sauerstoff (in Prozent der Sättigung) sowie pH bei den Meßpunkten 3-7, die außer Punkt 3 alle im Molendreieck liegen (Abb. 1). Uber ein halbes Jahr wurden monatliche Proben synchron bei Niedrigwasser genommen (Abb. 4). Die Wassertemperaturen zeigen im Molendreieck (Punkt 4-7) übereinstimmend im Winter etwas niedrigere, zum Sommer hin höhere Werte als im Strom vor der Mole (Punkt 3). Die Salzgehalte sind, wie zu erwarten war, im allgemeinen im Molendreieck etwas höher.

Die Sauerstoffgehalte des Wassers vor und hinter der Mole (Punkt 3 und 4) ähneln sich sehr, weil eine gute Verbindung durch das Lückensystem der Mole besteht; im allgemeinen liegen sie unter dem Sättigungswert, erst ab Mai nimmt der Sauerstoffgehalt zu. Im Watt des Molendreiecks liegen die Verhältnisse anders. Die sehr niedrigen Werte am 17. 2. und 9. 3. 1961 bei Punkt 5 und 6 bzw. 7 von 44-67, bzw. 79\% der Sättigung sind durch Nebel an diesen Tagen bedingt. Im übrigen ist die Sauerstoffproduktion in den Pfützen und flachen Stellen durch die Assimilation von Bodendiatomeen, in geringerem Maße auch durch andere Algen (z. B. Ulva) sehr intensiv, und es kommt zu bedeutenden Übersättigungen, die z. B. in einer Pfütze am Uferwerk (Punkt 7) bis zu 215\% (4. 5. 61) bzw. 232\% (17. 5. 61) betragen. Als Folge der Photosynthese steigen auch die pH-Werte beträchtlich an, besonders vom April ab.

Die gekennzeichneten Verhältnisse werden noch deutlicher, wenn man bei abgelaufenem Wasser die Veränderungen an verschiedenen Stellen verfolgt. Die Wasserproben wurden an einem warmen Sommertag vor und hinter der Nordmole (Punkt 3 und 4) sowie einer Wattenpfütze vor dem Uferwerk (Punkt 7) während drei Stunden genommen und die Werte miteinander verglichen (Abb. 5).

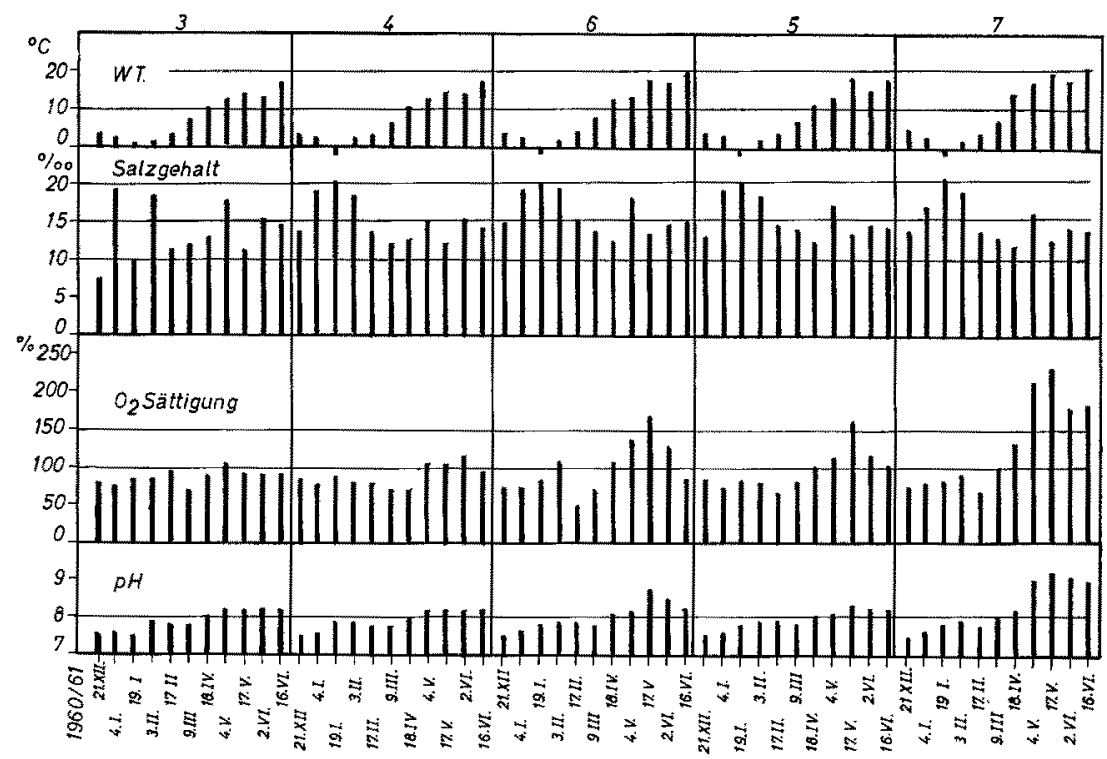

Abb. 4: Vergleich der Werte für Wassertemperatur, Salzgehalt, Sauerstoffgehalt (in Prozent der Sättigung) und pH für die Meßpunkte 3-7 in der Zeit vom Dezember 1960 bis Juni 1961. Die Proben wurden ein- oder zweimal im Monat bei Niedrigwasser genommen 
In der Pfütze stieg die Wassertemperatur nach drei Stunden von $18^{\circ} \mathrm{C}$ auf $25^{\circ} \mathrm{C}$ an, vor der Mole (3) nahm die Wassertemperatur nur von $16,2^{\circ} \mathrm{C}$ auf $20,8^{\circ} \mathrm{C}$ zu, hinter der Mole (4) von $17^{\circ} \mathrm{C}$ auf $20,8^{\circ} \mathrm{C}$. Wenn das Wasser über die Mole einläuft, findet ein Ausgleich statt. Um 9.15 Uhr betrug der Salzgehalt vor der Mole 13,09\%, in der Pfütze dagegen 22,48\%. Der Sauerstoffgehalt des Wassers im Strom (3) ändert

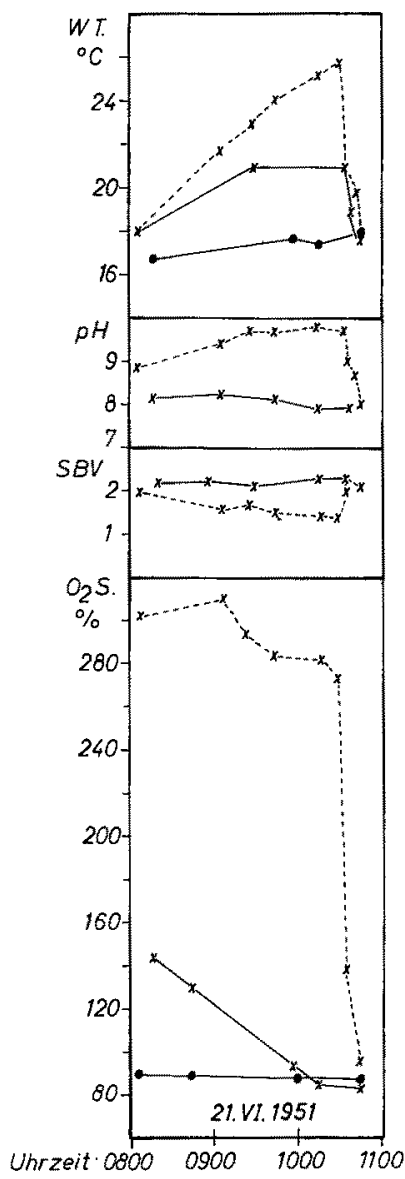

Abb. 5: Vergleich der Werte für Wassertemperatur, Säurebindungsvermögen (SBV), $\mathrm{pH}$ und Sauerstoffgehalt ( $\%$ der Sättigung) für die Meßpunkte 3- und $4 x-x$ (vor und hinter der Nordmole) und 7 (Wattenpfütze) $x \ldots \times \times$ während einer halben Tide. Tnw $8.44 \mathrm{Uhr}$

sich während der Untersuchungszeit nicht wesentlich. In der Pfütze (7) hingegen finden wir gleich bei Beginn der Probenentnahme eine sehr starke Sauerstoffübersättigung von $300 \%$ vor, die sich in der nächsten Stunde noch erhöht und dann wieder etwas abnimmt, was auf die Belichtungsverhältnisse zurüdkzuführen ist. Mit dem einlaufenden Flutwasser findet innerhalb weniger Minuten ein Rückgang auf $95 \%$ statt. Hinter der Mole, bei Punkt 4 haben wir zu Beginn eine Ubersättigung von $142 \%$. Da hier das auflaufende Wasser schon früher durch die Lücken zwischen den Steinen einströmt und 
sich mischt, nimmt die Ubersättigung stetig ab, so daß wir eine Stunde früher als in der Pfütze den Sauerstoffgehalt von $90 \%$ vorfinden.

Da die hohe Sauerstoffproduktion auf die Assimilation der Diatomeen zurückzuführen ist, ist es verständlich, daß sowohl der pH-Wert, wie auch die Werte für das Säurebindungsvermögen die entsprechenden Veränderungen zeigen: in der Pfütze

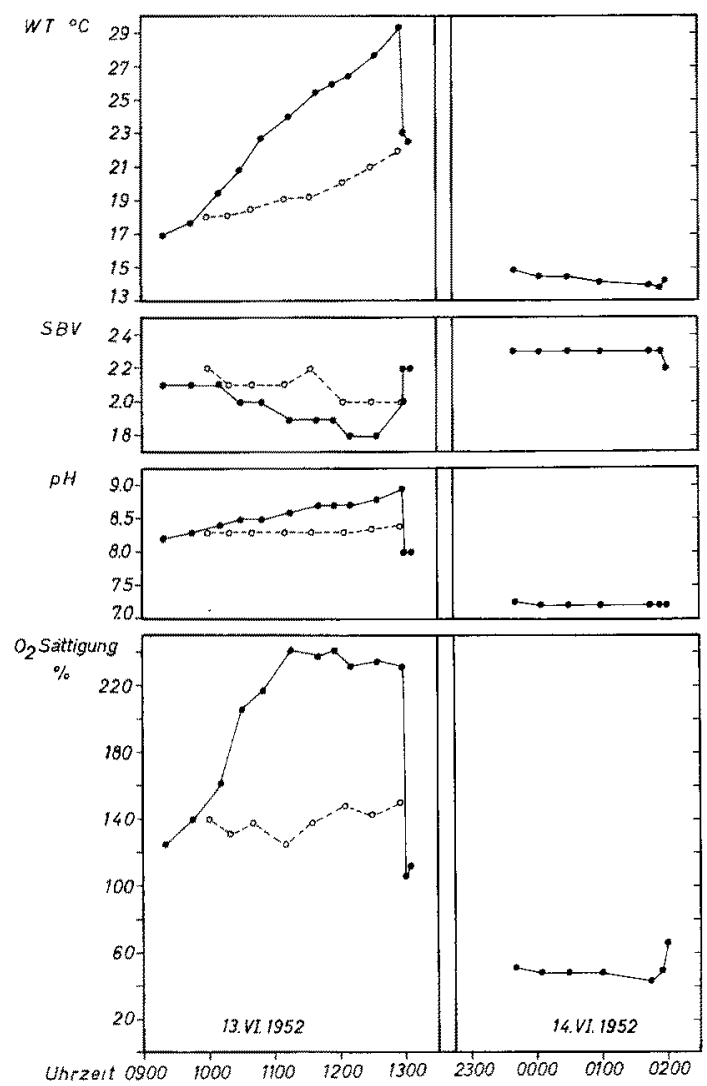

Abb. 6: Anderungen im Chemismus einer Wattenpfütze (Punkt 7) während zweier Tiden sowie bei Niedrigwasser am Tage (13.6.) und während der Nacht (14.6.). Die gestrichelte Kurve zeigt die Werte in der Wattenpfütze bei Verdunkelung (Abdecken durch eine Kiste) an. Tnw 11.20 und 23.54, Thw 16.54 Uhr (weitere Erklärungen s. Abb. 5)

steigt das $\mathrm{pH}$ sehr stark an, das SBV nimmt entsprechend ab. Bei den Punkten 3 und 4 verändern sich die Werte kaum, die Kurven laufen gleich (daher in Abb. 5 nur für Punkt 4 eingetragen. Durch die Aufmischung mit dem Flutwasser ändern sich $\mathrm{pH}$ und SBV genauso schnell wie die anderen Faktoren.

Die Wattenpfützen sind Kleinstgewässer, genauso wie Aquarien. Bei der Untersuchung des Chemismus in Aquarien konnten wir immer wieder feststellen, daß die Sauerstoffproduktion als Ausdruck der Assimilation den Anderungen äußerer Faktoren, z. B. der Beleuchtung außerordentlich schnell folgte (KüHL \& MANN 1951). 
Es mögen daher noch einige Reihen über die Tagesperiodizität in Wattenpfützen während der Trockenzeit folgen.

$\mathrm{Zu}$ diesem Zweck wurden am 13./14. Juli 1952 in der Pfütze am Uferwerk (Punkt 7) am. Tage (von 9.00 bis $13.00 \mathrm{Uhr}$ ) und in der Nacht (von 23.00 bis $2.00 \mathrm{Uhr}$ ) Proben während der Trodkenzeit genommen (Abb, 6). Die Tageskurve zeigt das schon bekannte Bild des Anstiegs der Wassertemperatur (von $17^{\circ}$ auf $29^{\circ} \mathrm{C}$ ), des Sauerstoffgebaltes (von 120 auf $230 \%$ der Sättigung), des $\mathrm{pH}$-Wertes (von 8,2 bis 9), die Abnahme des SBV (von 2,1 auf 1,8) und des plötzlichen Ausgleiches nach dem Einlaufen des Flutwassers. Der Salzgehalt war in der Pfütze bei Beginn 18,91\%, er stieg auf 20,16\% an und betrug nach der Aufmischung 15,58\%. In der Nacht findet dagegen keine Sauerstoffproduktion statt, es wird nur Sauerstoff verbraucht. Daher geht der Sauerstoffgehalt des Wassers auf 50-40\% der Sättigung zurüdk (in einem anderen Fall waren es sogar nur $27 \%$ ). Mit dem Einsetzen der Morgendämmerung stieg der Sauerstoffgehalt wieder an, die Probenentnahme wurde beendet. Dementsprechend verhalten sich auch $\mathrm{pH}$-Wert, der gleichbleibend bei 7,3-7,2 liegt, und das SBV mit 2,3. Die Wassertemperatur fällt von $15^{\circ}$ auf $14^{\circ} \mathrm{C}$ ab. Die Unterschiede im Salzgehalt sind $22,05 \%$ während der Trodkenzeit und 19,51\% nach dem Vollaufen der Pfütze.

In welch hohem Maße der Chemismus durch Wolkenbildung oder die Sonneneinstrahlung beeinflußt wird, soll durch einen Versuch in der gleichen Pfütze verdeutlicht werden. In die Mitte der Pfütze wurde eine unten offene und oben mit einem Dedkel versehene Kiste von den Ausmaßen $30 \times 30 \times 40 \mathrm{~cm}$ fest verankert. Gleichzeitig mit den Proben aus der Pfütze wurden auch solche aus der Kiste entnommen (ab 10.00 Uhr). Die Abbildung 6 (gestrichelte Kurve) bringt deutlich zum Ausdrudk, daß keine bedeutenden Änderungen im Sauerstoffgehalt auftreten, daß der $\mathrm{pH}$-Wert gleichbleibt und das SBV kaum abnimmt. Lediglich die Wassertemperatur steigt etwas an: von $17.2^{\circ}$ auf $21^{\circ} \mathrm{C}$. Es ergibt sich also das gleiche Bild wie in den Aquarien, wo

wir ja auch bei Verdunkelung sehr schnell die Assimilation bzw. die Sauerstoffproduktion mit allen Begleiterscheinungen herabsetzen konnten.

Der Ubergang der Nachtwerte zu den Tageswerten in der Pfütze (7) ist in Abbildung 7 dargestellt. Der Sauerstoffgehalt liegt in der Nacht bei 60-70\% der Sättigung, kurz nach Sonnenaufgang um 4.13 Uhr steigt die Sauerstoffkurve sehr schnell auf $210 \%$ der Sättigung an, um dann wieder bei auflaufendem Wasser schnell auf den "normalen" Sättigungswert abzusinken. Am Nachmittag (Abb. 7) wiederholt sich das gleiche, der $\mathrm{pH}$-Wert steigt von 7,3 auf 8,7 an, das SBV sinkt entsprechend ab.

Wenn die Elbe in kalren Wintern Eisgang hat, ist das Molendreieck wie auch das umgebende Watt mit Eisschollen bedeckt, die bei strengem Frost unter Umständen recht lange liegenbleiben können. Durch das einsickernde Wasser findet aber trotzdem noch eine ausreichende Belüftung des Lückensystems statt. Wir konnten unter den Eisschollen noch 60-70\% der Sauerstoffsättigung feststellen, was nicht bedeuten soll, daß der Sauerstoffgehalt nicht noch weiter absinken kann. Bei geringem Frost bedecken sich die Pfützen sehr schnell mit dünnen Eisschichten, die bei auflaufendem Wasser dann wieder verschwinden.

Einige Wirkungen der geschilderten Verhältnisse auf das biologische Geschehen sollen im folgenden ganz kurz dargestellt werden. Das Plankton besteht bei Cuxhaven zu einem bedeutenden Teil aus den Larvenformen von Benthosorganismen. Schon 
Thiemann (1937) wies auf Grund seiner Serien-Plankton-Untersuchungen während einer Tide in der Außenelbe auf bestimmte Maximumgebiete im Polyhalinikum beim FS Elbe 3 hin. Das Phytoplankton wurde im Zusammenhang mit verschiedenen chemischen Faktoren von Bursche, KüHL \& MANN (1958) während einer Tide einmal bei geringer Oberwassermenge (Sept. 1957:490 $\mathrm{m}^{3} / \mathrm{s}$ ) und bei starkem Abfluß (März 1957:

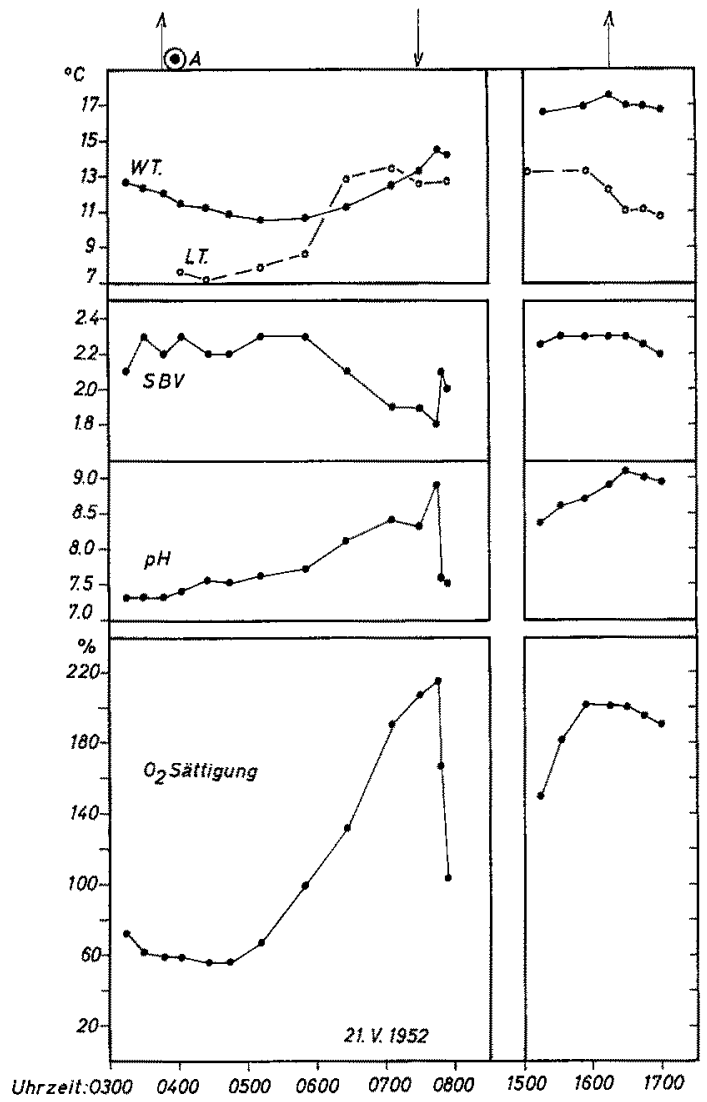

Abb. 7: Anderung im Chemismus einer Wattenpfütze (Punkt 7) bei Niedrigwasser und Sonnenaufgang (4.13 Uhr, durch A gekennzeichnet) Pfeil nach oben zeigend: Wasser läuft $a b$, Pfeil nach unten zeigend: Wasser läuft über die Nordmole. $L T=$ Luftemperatur

$\left.1818 \mathrm{~m}^{3} / \mathrm{s}\right)$. Erwartungsgemäß war der Salzgehalt im September mit max. 20\% höher als im März mit max. 12\%. Dementsprechend bestand das Septemberplankton nach Arten- und Individuenzahl vorwiegend aus marinen Phytoplanktern (zumeist Diatomeen). Im März war die Menge des marinen Phytoplanktons erheblich geringer. Beim Süßwasserplankton waren bezüglich der Artenzahl keine deutlichen Unterschiede zu bemerken, im März war jedoch der Anteil der toten Süßwasser-Phytoplankter im Verhältnis höher.

Mit zunehmendem Salzgehalt bei auflaufendem Wasser erhöht sich auch der Anteil der marinen Phytoplankter, und die Zahl der Süßwasserformen wird geringer, 
ebenso wie der Detritus bzw. Seston und Trübung. In ähnlicher Weise verändert sich auch das Zooplankton: mit zunehmendem Salzgehalt bei auflaufendem Wasser nimmt die Menge der Zooplankter zu, was besonders deutlich bei einigen in großer Zahl auftretenden Planktonformen wird, z. B. Spionidenlarven, Balanidenlarven und Cope-

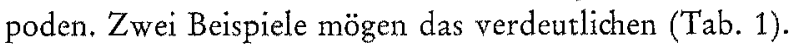

Tabelle 1

Prozentualer Anteil der Zooplankter

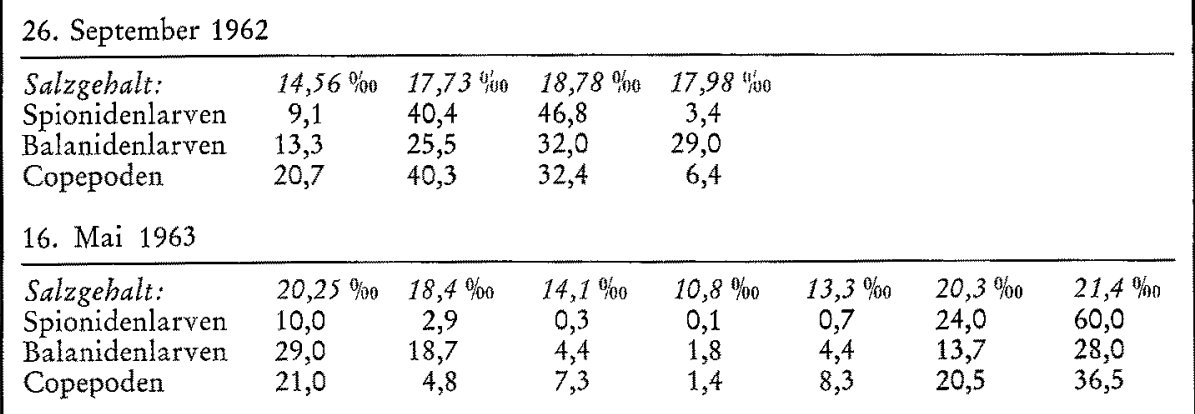

Bei stärkeren westlichen Winden werden im stärker salzhaltigen Wasser die Larven von Coelenteraten, Echinodermen (Asterias, Ophiuren, Echinocardium), Phoronis u. a. nach Cuxhaven verfrachtet, das eigentliche Siedlungsgebiet dieser Formen liegt jedoch unterhalb Cuxhavens, die Larven gehen daher vermutlich größtenteils zugrunde, auch wenn sie in den Fängen noch sehr wenig geschädigt erscheinen. Es genügt jedoch oft nur eine Erhöhung des Salzgehaltes um wenige Promille, damit sich die Larven entsprechend ihrer ökologischen Valenz weiterentwickeln können, es kommt dann zu einer Bestandsbildung. Diese Salzgehaltserhöhung kann eintreten, wenn, wie wir oben sahen, die Abflußmenge der Elbe für längere Zeit niedrig war, es also im Einzugsgebiet der Elbe längere Zeit nicht geregnet hat.

An wenigen Beispielen soll das erläutert werden. Die Anthomeduse Sarsia tubulosa kommt im Cuxhavener Plankton vom Winter bis Frühsommer regelmäßig in verschiedenen Altersstadien vor. Im Molendreieck konnten auch die Polypen Coryne sarsi bei Punkt 7 („Polypenpfütze“) gefunden werden, aber nur dann, wenn der Salzgehalt eine gewisse Höhe, mindestens in der zweiten Jahreshälfte, hatte (KüHL 1962).

Die $15 \%$-Linie muß überstiegen und die $20 \%$-Linie erreicht werden. Das war z. B. in den Jahren 1949 bis 1953 der Fall. In diesen Jahren traten die Polypen in wechselnden Mengen auf. Von 1954 bis 1958 waren keine Corynen vorhanden. Wie die Abbildung 2 zeigt, war in diesen Jahren als Folge eines größeren Abflusses der Elbe der Salzgehalt sehr niedrig. Erst im Jahre 1959 erschienen die Polypen wieder, als der Salzgehalt erneut eine größere Höhe erreicht hatte. Während durch die Wassertemperatur das Auftreten von Polypen und Medusen bestimmt wird, entscheidet der Salzgehalt in unserem Gebiet, ob die Polypen vorkommen oder nicht.

Auch die Stärke des Befalls durch die Holzbohrmuschel Teredo navalis L. in den hölzernen Hafenbauwerken und Holzschiffen wird durch die Höhe des Salzgehaltes 
bestimmt. Als in den Jahren 1947-49 der Salzgehalt infolge geringer Wasserführung recht hoch war, konnte ein sehr starker Befall festgestellt werden (KüHL 1957/58). Mit dem Absinken des Salzgehaltes von 1954-58 war Teredo nur vereinzelt anzutreffen und dann auch nur in den salzreicheren tieferen Wasserschichten. Ebenfalls erst im Jahre 1959 konnte wieder ein stärkerer Befall beobachtet werden. Die propagative Salzgehaltsgrenze dürte für Teredo navalis bei $15 \%$ liegen, während der vegetative Bereich bei $9 \%$ endet. Auf ausgelegten Platten wurden in Jahren mit höherem Salzgehalt bei Cuxhaven an der "Alten Liebe" Organismen beobachtet, die gewöhnlich im salzreicheren Polyhalinikum anzutreffen sind, z. B. Clava multicornis, Balanus crenatus, juvenile Asterias rubens. Lamellidoris bilamellata wurde häufiger auch mit Laichbändern angetroffen.

Im Molendreieck ist der Salzgehalt, wie oben gesagt, immer um einige Promille höher als in der Elbe, innerhalb des Molendreiecks ist er an der Westmole längs der Rinne (Abb. 1, Punkt 8) am höchsten. Diese Erhöhung genügte, daß sich von 1946-53 eine Population von Asterias rubens bildete. Die Seesterne erreichten aber nur eine Armlänge von 3-6 cm und wurden nicht fertil. Die Strandkrabbe Carcinus maenas kommt in größerer Zahl im Molendreieck und in der Umgebung vor, es findet auch die Kopula statt, es konnten aber keine Weibchen mit Eiern gefunden werden. Die Megalopen und jungen Krabben bevölkern das Gebiet aber in riesigen Mengen. Bemerkenswert war uiber zwei Jahre (1951/52) das Vorkommen von kleinen Eupagurus bernhardus in einer Pfütze an der Nordmole (Nähe Punkt 5). Die Einsiedler saßen in Litorinagehäusen. Nach zwei Jahren verschwanden die Einsiedler wieder. Zoeen von Einsiedlern sind im Plankton an der "Alten Liebe“ recht selten.

Unter den abiotischen Faktoren beeinflußt, wie wir sahen, der Salzgehalt mit seinen bedeutenden Schwankungen weitgehend die Lebensgemeinschaften in der Elbmündung. Mit der Flut werden im Plankton die Larvenstadien herangeführt, von denen viele ihre größte Wohndichte unterhalb von Cuxhaven beim Feuerschiff Elbe 3 haben (Thiemann 1934, KüHz \& Mann 1953). Werden nun im untersuchten Gebiet die geeigneten Bedingungen für die Metamorphose und den Ubergang zum Bodenleben vorgefunden, so kann es zu einer mehr oder weniger lang andauernden Ansiedlung kommen. Die günstigen Bedingungen brauchen für eine Reihe von Arten nur für die Übergangszeit vorzuherrschen, da die Widerstandsfähigkeit mit zunehmendem Alter wächst (vegetativer Temperatur- und Salzgehaltsbereich, Runnström 1936). Diese Bedingungen werden durch die Wasserführung der Elbe geschaffen. Da sie zumeist langfristig einwirken, beeinflussen sie die Lebensgemeinschaften am nachhaltigsten.

Ein sehr starker Wechsel der abiotischen Faktoren kann in kleinen Wasserlachen des Wattes auftreten, wenn durch bestimmte Wetterbedingungen extremale Verhältnisse hervorgerufen werden, wie schnelles Steigen und Fallen von Wassertemperatur und Salzgehalt, oder als Folge intensiver Assimilation der Algen starke Sauerstofferhöhung, Anstieg des pH-Wertes usw., in der Nacht durch Dissimilation ein Sauerstoffrïckgang. Die Veränderungen treten sehr schnell auf und werden bei einlaufendem Flutwasser plötzlich wieder ausgeglichen (NıCOL 1935, KüHL 1952).

Niedrige winterliche Wassertemperaturen mit längerer Eisbedeckung brauchen im Watt bzw. Molendreieck keineswegs katastrophale Folgen zu haben, wie der lange Winter 1962/63 erwiesen hat. Nach monatelanger Eisbedeckung waren die meisten 
Wattorganismen am Leben geblieben (z. B. Coropbium, Balaniden, außer Elminizs, Nereiden, Polydora, Heteromastus, Mytilus), während die Miesmuscheln am Bollwerk „Alte Liebe ${ }^{\text {"c }}$ an den Pfählen vollständig ausgewintert waren, auch ohne den Abrieb durch Eisschollen.

Die bedeutenden Schwankungen des Salzgehaltes und anderer chemischer Faktoren, die damit in mittelbarem oder unmittelbarem Zusammenhang stehen, wirken stark limitierend (Remane 1940, 1959, Remane \& Schlieper 1958, Kinne 1956). Wenn bei dem oben angeführten Vergleich zwischen der Elbe und einer Ostseebucht die Artenzahl im Elbebereich wesentlich geringer war, so dïrfte außer der Strömung und anderen Bedingungen hauptsächlich die große Schwankungsbreite des Salzgehaltes, die bei Cuxhaven an der Oberfläche von 3-29\% reichen kann (KüHL 1953-63), der Grund sein. Die Verarmungszone liegt ja auch im Bereich des "Salzgehaltssprunges", der schnellen Anderung des Salzgehaltes, in dem auch die Schwankungen am größten sind (KüHL \& ManN 1953, Caspers 1948, 1958, Schulz 1961, „extreme Lebensstätte" nach Thienemann 1939, Lucht 1953).

Die Organismen, die dauernd im Gebiet vorkommen, haben die Fähigkeit, den Wechsel der Milieubedingungen zu überstehen, sei es, daß sie die ungünstigen Perioden durch Verschluß ihrer Gehäuse oder Wohnröhren überstehen (Balaniden, Mytilus, Teredo, Litorina), durch Eingraben, Herabsetzen des Stoffwechsels oder durch Osmoregulation u. a. überwinden. Das Vorhandensein oder Fehlen bestimmter Arten in verschiedenen Jahren, auf das bereits KIRCHENPAuER (1862) hinwies, ist hauptsächlich durch länger andauernde Anderung im Salzgehalt bedingt.

Trotz des großen Schwankungsbereiches des Salzgehaltes hat sich die Einteilung in haline Zonen nach REDEkE, wie sie jetzt im „Venedig-System" vorliegt (CASPERS 1959), bewährt, wenn man von dem Einteilungssystem nicht mehr verlangt, als es geben kann.

\section{ZUSAMMENFASSUNG}

1. Die Veränderungen der abiotischen Faktoren, die schon den frühen Untersuchern bekannt waren, werden besprochen.

2. Langfristige Änderungen des Salzgehaltes werden durch die Abflußmenge der Elbe bedingt.

3. Kurzfristige Anderungen der abiotischen Faktoren bringen die Gezeiten; ihr Ausmaß wird weitgehend von den Wetterbedingungen beeinflußt. Im Watt sind während der Ebbe je nach den Außenbedingungen starke Veränderungen im Chemismus festzustellen, deren Ursache z. T. auf die Photosynthese der Diatomeen zurückzuführen ist. Messungen selbst an nahe beieinander gelegenen Punkten bringen schon große Unterschiede der abiotischen Faktoren.

4. In einem durch Molen abgegrenzten Gebiet an der "Alten Liebe“ ist der Salzgehalt immer etwas höher als Folge seiner topographischen Besonderheit.

5. Die Wirkungen der Veränderungen abiotischer Faktoren auf das biologische Geschehen werden an einigen Beispielen erläutert, z. B. das Erscheinen und Verschwinden bestimmter Arten in einzelnen Jahren in Abhängigkeit von der Abflußmenge der Elbe und im Zusammenhang damit von der Höhe des Salzgehaltes. 


\section{ZITIERTE LITERATUR}

Bursche, E. M., KühL, H. \& ManN, H., 1958a. Hydrochemische Faktoren und Phytoplankton während einer Tide in der Elbmündung. Gewäss. Abwäss. 8, 13-39.

- 1958b. Hydrochemie und Phytoplankton in der Unterelbe. Veröff. Inst. Meeresforsch. Bremerhaven 5, 165-192.

CASPERs, H., 1948. Ơologische Untersuchungen über die Wattentierwelt im Elbeaestuar. Verh. dtsch. zool. Ges. Kiel 1948, 350-359.

- 1952. Bodengreiferuntersuchungen über die Tierwelt in der Fahrrinne der Unterelbe und Vormündungsgebiet der Nordsee. Verh. dtsch. zool. Ges. Wilhelmshaven 1951, 404-418.

- 1958. Biologie der Brackwasserzonen im Elbeaestuar. Verh. int. Ver. Limnol. 13, 686-698.

- 1959. Vorschläge einer Bradkwassernomenklatur (The Venice System). Int. Rev. Hydrobiol. 44, 313-315.

Dakt, F., 1892. Untersuchungen über die Thierwelt der Unterelbe. Comm. wriss. Unters. dtsch. Meere 3, 149-185.

Friedrich, H., 1952. Über neuere Gesichtspunkte zur Physiologie der Biocoenosen. Veröff. Inst. Meeresforsch. Bremerbaven 1, 225-231.

Krnne, O., 1956. Uber Temperatur und Salzgehalt und ihre physiologisch-biologische Bedeutung. Biol. Zbl. 75, 314-327.

- 1956b. Über den Wert kombinierter Untersuchungen (im Biotop und im Zuchtversuch) für die ökologische Analyse. Naturwissenscbaften 43, 8-9.

Krrchenpauer, H. G., 1862. Die Seetonnen der Elbmündung. Ein Beitrag zur Thier- und Pflanzentopographie. Abh. naturw. Ver. Hamburg. IV. Abt. 3, 1-59.

KüHL, H., 1953-63. Oberflächen-Wassertemperaturen und Salzgehalt an der "Alten Liebe" bei Cuxhaven in Abhängigkeit von Jahreszeit, Windrichtung und Pegelstand (1947-52. Veröff. Inst. Kïst.- u. Binnenfisch. 3/1953. Für die Jahre 1953-62: Ebenda 11/1955, 13/1956, 14/ 1956, 15/1957, 18/1958, 19/1959, 21/1960, 23/1961, 24/1962, 26/1963.

- 1952. Über die Hydrographie von Wattenpfützen. Helgol. Wiss. Meeresunters. 4, 101-106.

- 1957/58. Das Auftreten mariner Holzschädlinge (Bohrmuscheln und Bohrkrebse) in Flußmündungen und Häfen in Abhängigkeit von den Wasserverhältnissen. In: Arbeiten d. Deutsch. Fischerei-Verbandes, Heft 10, 1958, und Das Fischerblatt 12/1957, 1/1958.

- 1957. G. H. Kirchenpauers Untersuchung der Elbmündung. Kosmos, Stuttgart 53, 104-108.

- 1962. Die Hydromedusen der Elbmündung. Abh. naturw. Ver. Hamburg. NF. 1961, 6, $209-232$.

KüHL, H. \& ManN, H., 1953. Beiträge zur Hydrochemie der Unterelbe. Veröff. Inst. Meeresforsch. Bremerbaven 2, 236-268.

- 1962. Über das Zooplankton der Unterelbe. Veröff. Inst. Meeresforsch. Bremerbaven 8, 53-70.

- 1951. Uber die periodischen Anderungen im Chemismus von Seewasseraquarien. Verb. dtsch. zool. Ges. 1951, 378-385.

LuchT, F, 1953. Hydrographische Untersuchungen der Brackwasserzone der Elbe. Dtsch. bydrogr. Z. 6, 18-32.

LüNEBURG, H., 1939. Hydrochemische Untersuchungen der Elbmündung mittels Elektrokolorimeter. Arch. dtsch. Seewarte 59, 1-27.

Nicon, E., 1935. The ecology of a salt marsh. J. Mar. biol. Ass. U. K. 20, 203-261.

REDEKE, H. C., 1922. Zur Biologie der niederländischen Brackwassertypen. (Ein Beitrag zur regionalen Limnologie.) Bjdr. Dierk. Amsterdam 22, 329-335.

- 1933. Über den jetzigen Stand unserer Kenntnisse der Flora und Fauna des Brackwassers. Verb. int. Ver. Limnol. 6, 46-61.

Remane, A, 1940. Einführung in die zoologische Okologie der Nord- und Ostsee. Tierwelt N.- u. Ostsee 1, 1-238.

- 1959. Regionale Verschiedenheiten der Lebewesen gegenüber dem Salzgehalt und ihre Bedeutung für die Brackwasser-Einteilung. Archo Oceanogr. Limnol. XI. Suppl., 35-46.

- \& SChlieper, C., 1958. Die Biologie des Bradkwassers. In: Die Binnengewässer, Bd 22. Schweizerbart, Stuttgart, $348 \mathrm{pp}$. 
RunNström, S., 1936. Die Anpassung der Fortpflanzung und Entwicklung mariner Tiere an die Temperaturverhältnisse verschiedener Verbreitungsgebiete. Bergens Mus. Aarb. Naturvidensk. R. 3.

Schulz, H., 1961. Qualitative und quantitative Planktonuntersuchungen im Elbe-Aestuar. Arch. Hydrobiol. Suppl. XXVI/1, 5-105.

Thiemann, K., 1934. Das Plankton der Flußmündungen. Meteor 12, 199-273.

Thienemann, A., 1939. Grundzüge einer allgemeinen OKoologie. Arcb. Hydrobiol. 35, 267-285.

\section{Diskussion im Anschluß an den Vortrag KüHL}

LüNEBuRG: Warum gefriert das Sediment des Wattes nicht in die Eisschollen hinein?

KüHL: Die Beobachtung, daß Eisschollen den unter ihnen liegenden Wattboden einfrieren und beim Aufschwimmen dann mitnehmen, konnte of gemacht werden. An einer Eisscholle wurde sogar einmal eine fünffache Schichtung festgestellt. Es brauchen aber nicht alle Eisschollen auf dem Wattboden festzufrieren, sie schwimmen dann bei Flut schnell auf, ohne Sediment mitzunehmen; oder unter einer Decke von Eisschollen befinden sich Lücken, in die Seewasser eindringen kann. Zwischen und unter den Eisschollen haben wir - oft mit Schwierigkeiten Wattboden und Wasserproben entnommen, wobei uns besonders die Temperatur- und Sauerstoffverhältnisse interessierten. Aus Wattproben, die monatelang von Eisschollen bedeckt waren, ohne aber angefroren zu sein, kamen im Laboratorium nach kurzer Zeit Würmer und Krebse in großer Zahl lebend zur Oberfläche.

LÜNEBURG: Wie groß ist der Abwassereinfluß?

KüHL: Das Abwasser kommt bei Ebbstrom an der „Alten Liebe“ vorbei; in den "Plankton"Proben findet man dann sehr viel Papier, Schmutz und wachsähnliche Reste der häuslichen Abwässer. Das Abwasser fließt als 5 bis $10 \mathrm{~m}$ breite Fahne flußabwärts und wird später durch Turbulenz verwirbelt. Im ganzen gesehen dürtte der direkte Einfluß nur gering sein. 